Proceedings of the Edinburgh Mathematical Society (2008) 51, 45-56 (C)

DOI:10.1017/S0013091506000277 Printed in the United Kingdom

\title{
SOME NEW IMMERSION RESULTS FOR COMPLEX PROJECTIVE SPACE
}

\author{
DONALD M. DAVIS \\ Department of Mathematics, Lehigh University, \\ Bethlehem, PA 18015, USA (dmd1@lehigh.edu)
}

(Received 21 February 2006)

\begin{abstract}
We prove the following two new optimal immersion results for complex projective space. First, if $n \equiv 3 \bmod 8$ but $n \not \equiv 3 \bmod 64$, and $\alpha(n)=7$, then $C P^{n}$ can be immersed in $\mathbb{R}^{4 n-14}$. Second, if $n$ is even and $\alpha(n)=3$, then $C P^{n}$ can be immersed in $\mathbb{R}^{4 n-4}$. Here $\alpha(n)$ denotes the number of 1 s in the binary expansion of $n$. The first contradicts a result of Crabb, which said that such an immersion does not exist, apparently due to an arithmetical mistake. We combine Crabb's method with that developed by the author and Mahowald.
\end{abstract}

Keywords: immersions; complex projective space; obstruction theory

2000 Mathematics subject classification: Primary 57R42; 55S35

\section{Main theorems}

We prove the following two new optimal immersion results for $2 n$-dimensional complex projective space $C P^{n}$.

Theorem 1.1. If $n \equiv 3 \bmod 8$ and $n \not \equiv 3 \bmod 64$, and $\alpha(n)=7$, then $C P^{n}$ can be immersed in $\mathbb{R}^{4 n-14}$.

Theorem 1.2. If $n$ is even and $\alpha(n)=3$, then $C P^{n}$ can be immersed in $\mathbb{R}^{4 n-4}$.

Here and throughout, $\alpha(n)$ denotes the number of $1 \mathrm{~s}$ in the binary expansion of $n$. Theorem 1.1 contradicts a result of Crabb [3]. In $\S 2$, we prove Theorem 1.1 by an adaptation of Crabb's argument, and point out what we believe to be his mistake, apparently in the arithmetic. We prove Theorem 1.2 in $\S 3$.

We now summarize what we feel to be the status of the immersion question for $C P^{n}$. In addition to incorporating the two new immersion results above, we list as unresolved one immersion result claimed by Crabb. We will discuss our reason for doing so in $\S 4$. Despite our feeling that two of Crabb's many results are flawed, we feel that his overall approach, combining K-theory with obstruction theory, is sound; we have checked the details of his immersion results cited in Theorem 1.7. Now we begin our summary.

There are three families of results that apply to all values of $\alpha(n)$. All known nonimmersion results follow from the first two. 
Table 1. Immersions and non-immersions when $n$ is even (see Theorem 1.6)

\begin{tabular}{rcrr}
$\alpha(n)$ & $d$ & $e$ \\
\hline 2 & & 2 & 3 \\
3 & 4 & 5 \\
4 & $n \neq 10(\bmod 16)$ & 7 & 8 \\
4 & 7 & 9 \\
5 & 9 & 10 \\
6 & & 10 & 11 \\
7 & & 11 & 13 \\
8 & $n \neq 1022(32)$ and $\not \equiv 132(256)$ & 14 & 16 \\
8 & $n \equiv 22(32)$ or $\equiv 132(256)$ & 14 & 17 \\
$>8$ & & 14 & \\
\hline
\end{tabular}

Theorem 1.3 (Sanderson and Schwarzenberger [13]). $C P^{n}$ cannot be immersed in $\mathbb{R}^{4 n-2 \alpha(n)+\varepsilon}$, where

$$
\varepsilon= \begin{cases}0 & \text { if } n \text { is even and } \alpha(n) \equiv 1(\bmod 4) \\ 1 & \text { if } n \text { is even and } \alpha(n) \equiv 2,3(\bmod 4) \\ -1 & \text { otherwise }\end{cases}
$$

Theorem 1.4 (Sigrist and Suter [14]). If $C P^{n}$ immerses in $\mathbb{R}^{4 n-2 \alpha(n)}$, then

$$
\nu\left(c_{\alpha(n)-1}\right)=\nu\left(c_{\alpha(n)}\right)<\nu\left(c_{i}\right) \text { for all } i<\alpha(n)-1,
$$

where $\sum c_{i} t^{i}=((\log (1+t)) / t)^{2 n+1-\alpha(n)}$.

Here and throughout, $\nu(-)$ denotes the exponent of 2 dividing an integer. The specific results obtainable from Theorem 1.4 were determined for $\alpha(n) \leqslant 5$ in [14] and for $\alpha(n)=6$ and 7 (with a mistake when $\alpha(n)=7$ ) in $[\mathbf{3}]$. In $\S 5$, we derive these for $\alpha(n)=8$; the results have been incorporated into Tables 1 and 2 .

For large values of $\alpha(n)$, the best immersion results are obtained in the following embedding theorem, which relied on earlier results of Milgram.

Theorem 1.5 (Mukherjee [11]). $C P^{n}$ can be embedded in $\mathbb{R}^{4 n-\alpha(n)}$, and, if $n>1$ is odd, $C P^{n}$ can be embedded in $\mathbb{R}^{4 n-\alpha(n)-1}$.

For small $\alpha(n)$, better immersion results are obtained by Davis and Mahowald [7] and Crabb [3]. Crabb did not consider even values of $n$, and so, when $n$ is even, the immersions are from [7] and Theorem 1.2, and the non-immersions are from [13] (stated here as Theorem 1.3) and from [14] (stated here as Theorem 1.4).

Theorem 1.6. If $n$ is even, then $C P^{n}$ immerses in $\mathbb{R}^{4 n-d}$ and does not immerse in $\mathbb{R}^{4 n-e}$, where $d$ and $e$ are given in Table 1 . 
Table 2. Immersions and non-immersions when $n$ is odd (see Theorem 1.7)

\begin{tabular}{clrcrc}
\hline$\alpha(n)$ & condition & $d$ & reference & $e$ & reference \\
\hline 2 & & 3 & {$[\mathbf{9}]$} & 4 & {$[\mathbf{1 4}]$} \\
3 & $n \equiv 1(\bmod 4)$ & 5 & {$[\mathbf{1 2}]$} & 6 & {$[\mathbf{1 4}]$} \\
3 & $n \equiv 3(\bmod 4)$ & 6 & {$[\mathbf{1 6}]$} & 7 & {$[\mathbf{1 3}]$} \\
4 & $n \neq 7(\bmod 8)$ & 7 & {$[\mathbf{7}]$} & 8 & {$[\mathbf{1 4}]$} \\
4 & $n \equiv 7(\bmod 8)$ & 8 & {$[\mathbf{3}]$} & 9 & {$[\mathbf{1 3}]$} \\
5 & $n \equiv 1(\bmod 4)$ & 9 & {$[\mathbf{7}]$} & 11 & {$[\mathbf{1 3}]$} \\
5 & $n \equiv 3(\bmod 8)$ & 9 & {$[\mathbf{7}]$} & 10 & {$[\mathbf{1 4}]$} \\
5 & $n \equiv 7(\bmod 8)$ & 10 & {$[\mathbf{3}]$} & 11 & {$[\mathbf{1 3}]$} \\
6 & $n \equiv 1(\bmod 4)$ & 11 & {$[\mathbf{7}]$} & 12 & {$[\mathbf{1 4}]$} \\
6 & $n \equiv 3(\bmod 16)$ & 11 & {$[\mathbf{7}]$} & 12 & {$[\mathbf{1 4}]$} \\
6 & $n \equiv 11(\bmod 16)$ & 12 & {$[\mathbf{3}]$} & 13 & {$[\mathbf{1 3}]$} \\
6 & $n \equiv 7(\bmod 8)$ & 12 & {$[\mathbf{3}]$} & 13 & {$[\mathbf{1 3}]$} \\
7 & $n \equiv 1(\bmod 4)$ & 13 & {$[\mathbf{7}]$} & 14 & {$[\mathbf{1 4}]$} \\
7 & $n \equiv 3(\bmod 64)$ & 13 & {$[\mathbf{7}]$} & 14 & {$[\mathbf{1 4}]$} \\
7 & $n \equiv 3(8), \neq 3(64)$ & 14 & Theorem 1.1 & 15 & {$[\mathbf{1 3}]$} \\
7 & $n \equiv 7(\bmod 8)$ & 14 & {$[\mathbf{3}]$} & 15 & {$[\mathbf{1 3}]$} \\
8 & $n \neq \equiv 15(16)$ and $\not \equiv 37(64)$ & 15 & {$[\mathbf{7}]$} & 16 & {$[\mathbf{1 4}]$} \\
8 & $n \equiv 15(16)$ or $\equiv 37(64)$ & 15 & {$[\mathbf{7}]$} & 17 & {$[\mathbf{1 3}]$} \\
$>8$ & & 15 & {$[\mathbf{7}]$} & & \\
\hline
\end{tabular}

Thus, when $n$ is even, the only unresolved case for $\alpha(n) \leqslant 6$ occurs when $\alpha(n)=4$ and $n \equiv 10 \bmod 16$.

We believe that the tabulation of results and earliest proofs given in Table 2 is accurate when $n$ is odd. Note that the case discussed in Proposition 4.1 is the only unresolved case when $n$ is odd and $\alpha(n) \leqslant 7$.

Theorem 1.7. If $n$ is odd, then $C P^{n}$ immerses in $\mathbb{R}^{4 n-d}$ and does not immerse in $\mathbb{R}^{4 n-e}$, where $d$ and $e$ are given in Table 2 .

\section{Proof of Theorem 1.1}

In this section we prove Theorem 1.1 and describe what we believe was Crabb's mistake when he asserted a non-immersion in this situation.

Let $H_{n}^{\mathbb{C}}$ denote the Hopf bundle over $C P^{n}$. It is standard that the immersion in Theorem 1.1 is equivalent to showing that the stable normal bundle $-(n+1) H_{n}^{\mathbb{C}}$ is stably equivalent to a bundle of dimension $2 n-14$. We let $n=8 p+3$ with $\alpha(p)=5$. In $[\mathbf{3}, \S 3$, especially (3.2)], Crabb showed that a necessary condition for the immersion is that, if $\lambda(T)=\left(\sinh ^{-1}(\sqrt{T}) / \sqrt{T}\right)^{2}$, and $\left(\lambda^{8 p}\right)_{i}$ denotes the coefficient of $T^{i}$ in $\lambda(T)^{8 p}$, then there exists an integer $e$ such that $e\left(\lambda^{8 p}\right)_{3} \equiv 64 \bmod 128, e\left(\lambda^{8 p}\right)_{2} \equiv 0 \bmod 32$, 
$e\left(\lambda^{8 p}\right)_{1} \equiv 0 \bmod 8$ and $e\left(\lambda^{8 p}\right)_{0} \equiv 0 \bmod 2$. (For the reader wishing to compare with Crabb's notation, his $l=14, j=3$, and $k=4 p+1$.)

Working $\bmod \left(T^{4}\right)$, we have

$$
\lambda(T)=1-\frac{1}{3} T+\frac{8}{45} T^{2}-\frac{4}{35} T^{3},
$$

and

$$
\lambda(T)^{8}=1-\frac{8}{3} T+\frac{68}{15} T^{2}-\frac{1192}{189} T^{3} .
$$

We then have

$$
\lambda(T)^{8 p}=1+u_{1} 8 p T+u_{2} 4 p T^{2}+u_{3} 8 p T^{3},
$$

where each $u_{i}$ is an odd fraction. The first and last of Crabb's necessary conditions stated above require $e p \equiv 8 \bmod 16$ and $e \equiv 0 \bmod 2$. These (and the other conditions) can be satisfied if and only if $p \not \equiv 0 \bmod 8$. Crabb's Lemma 3.4 makes it clear that he believed that his conditions could not be satisfied in the cases which we address here.

Now we prove that the immersion exists when $p \not \equiv 0 \bmod 8$. We use modified Postnikov towers (MPTs), as introduced in [8] and employed in many papers, such as $[\mathbf{6}, \mathbf{7}]$ and, more recently, $[\mathbf{1 5}]$. We consider the lifting question

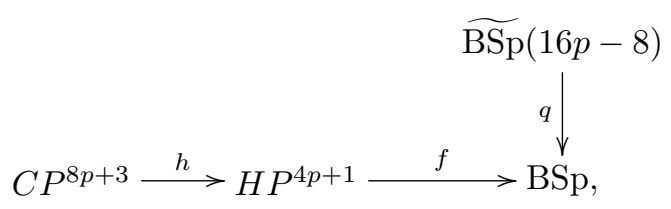

where $f$ classifies the stable bundle $-(4 p+2) H_{4 p+1}^{S p}$ over the quaternionic projective space. The space $\widetilde{\mathrm{BSp}}(m)$ is the classifying space for symplectic vector bundles of real geometric dimension $m$. It is the pull-back of $B O(m)$ and BSp over $B O$. We let

$$
\widetilde{\mathrm{BSp}}(16 p-8)=E_{8} \rightarrow E_{7} \rightarrow \cdots \rightarrow E_{1} \rightarrow \mathrm{BSp}
$$

denote the MPT through dimension $16 p+6$. In this range, the fibre of $q$ is the stable stunted real projective space $P_{16 p-8}=R P^{\infty} / R P^{16 p-9}$, whose homotopy groups in this range are displayed in [10, Table 8.9]. We reproduce them in Figure 1, indexed as $\pi_{*}\left(\Sigma P_{16 p-8}\right)$, which are their dimensions as $k$-invariants in the MPT.

The obstructions for lifting from $E_{i}$ to $E_{i+1}$ are $k$-invariants in $H^{j}\left(E_{i}\right)$ corresponding to dots in position $(j, i)$ of the diagram. All cohomology groups have coefficients in $\mathbb{Z} / 2$. The bulk of our work will be in proving the following result, which states that $f$ lifts to the fifth stage of the MPT.

Proposition 2.1. In (2.1), $f$ factors through a map $H P^{4 p+1} \stackrel{f_{5}}{\longrightarrow} E_{5}$.

Before giving the proof of Proposition 2.1, we use it to complete the proof of Theorem 1.1. Let $\ell=f_{5} \circ h: C P^{8 p+3} \rightarrow E_{5}$. To get $C P^{8 p+1}$ to lift to $\widetilde{\mathrm{BSp}}(16 p-8)$, we need only show that $\ell^{*}\left(k_{2}\right)=0$, where $k_{2} \in H^{16 p+2}\left(E_{5}\right)$ corresponds to the dot in position $(16 p+2,5)$. The diagonal line emanating from this dot suggests, and the computation 


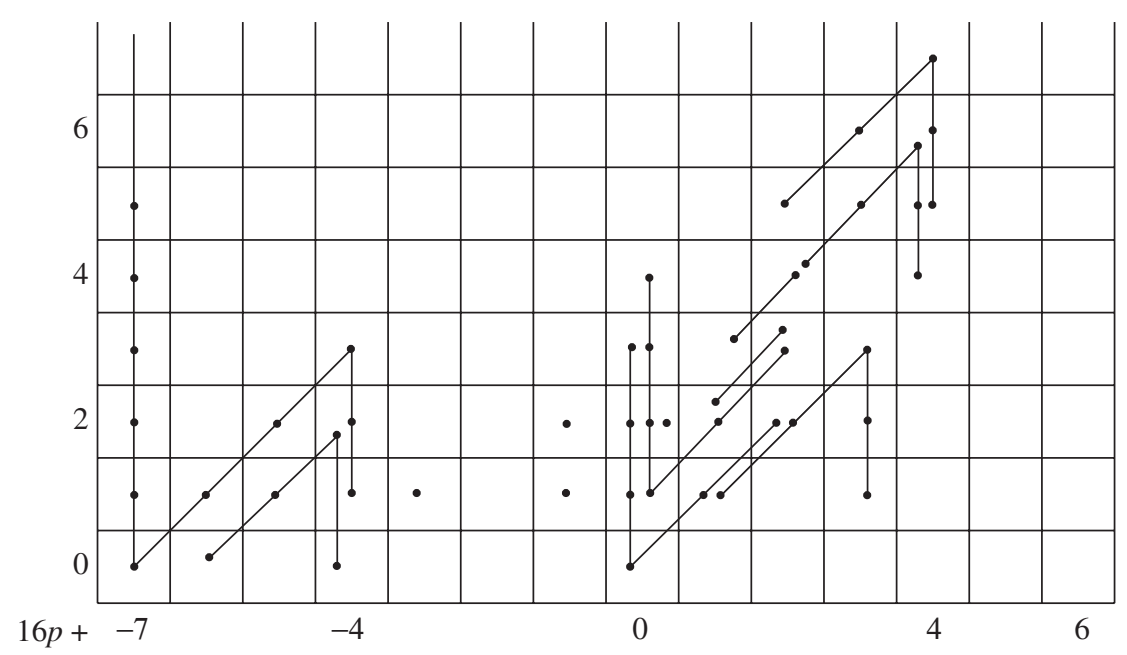

Figure 1. The Adams spectral sequence of $\Sigma P_{16 p-8}$.

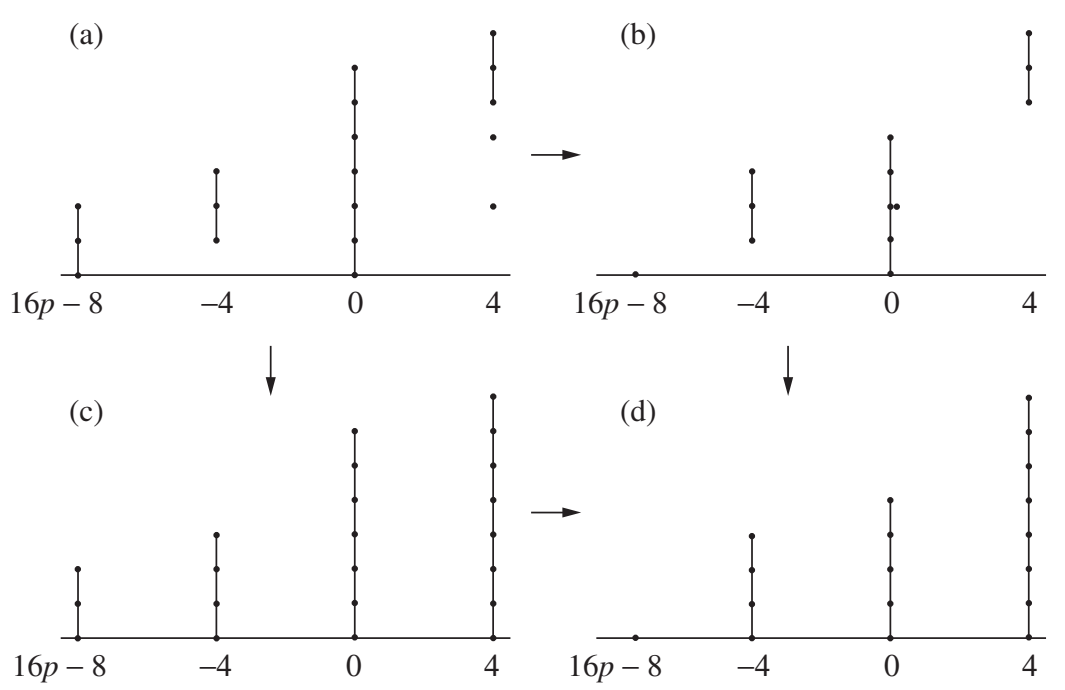

Figure 2. Possible obstructions for liftings in (2.2): (a) $\Sigma P_{16 p-11}$; (b) $\Sigma P_{16 p-9} ;$ (c) $\Sigma P_{16 p-11} \wedge$ bo; (d) $\Sigma P_{16 p-9} \wedge$ bo.

of the MPT proves, that there is a relation in $H^{*}\left(E_{5}\right)$ of the form $\mathrm{Sq}^{2} k_{2}+a k_{-7}=0$, where $k_{-7} \in H^{16 p-7}\left(E_{5}\right)$ corresponds to the dot at height 5 in the initial tower, and $a$ is a combination of Steenrod operations and Stiefel-Whitney classes acting on $k_{-7}$. Therefore, since $H^{16 p-7}\left(C P^{8 p+3}\right)=0$, we must have $\operatorname{Sq}^{2}\left(\ell^{*}\left(k_{2}\right)\right)=0$ in $H^{*}\left(C P^{8 p+3}\right)$. Since $\mathrm{Sq}^{2}$ acts injectively on $H^{16 p+2}\left(C P^{8 p+3}\right)$, this implies $\ell^{*}\left(k_{2}\right)=0$, and hence $C P^{8 p+1}$ lifts to $\widetilde{\mathrm{BSp}}(16 p-8)$.

By [3, Proposition 3.2], the $K O$-theoretic obstruction to extending this lifting over $C P^{8 p+2}$ is given by the conditions on $\lambda^{8 p}$ described above, which are satisfied under our 
hypotheses, and hence this $K O$-theoretic obstruction is 0 . The total obstruction to this extension lies in $\pi_{16 p+4}\left(\Sigma P_{16 p-8}\right) \approx \mathbb{Z} / 8 \oplus \mathbb{Z} / 8$, depicted in Table 1 , but in this case the total obstruction is entirely $K O$-theoretic, as described in [2, Proposition 4.6]. See also row 3 of [2, Table 4.1], which states explicitly that the kernel of reduction from total obstruction to $K O$-theoretic obstruction is 0 . From our viewpoint, the non- $K O$-theoretic obstructions are irregular classes such as the one in position $(16 p, 2)$ in Figure 1, which must be dealt with in the proof of Proposition 2.1.

Thus, $C P^{8 p+2}$ lifts to $\widehat{\mathrm{BSp}}(16 p-8)$. Since, by Figure $1, \pi_{16 p+6}\left(\Sigma P_{16 p-8}\right)=0$, this lifting extends over $C P^{8 p+3}$, as required for Theorem 1.1.

We complete the proof of Theorem 1.1 by proving Proposition 2.1. We will use the bo-primary classifying spaces $B^{\circ}(m)$ constructed in [6]. There is a map of fibrations through dimension $2 m-2$ :

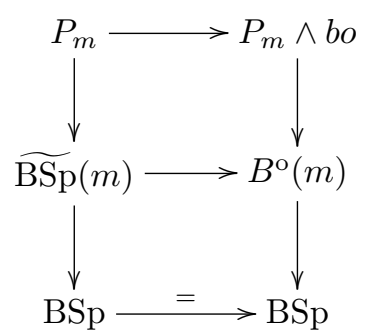

and there are natural maps of MPTs for these fibrations. We will consider the maps of MPTs for the following spaces over BSp:

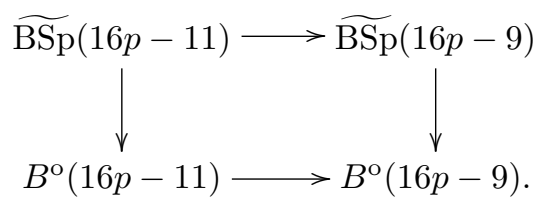

We depict in Figure 2 the portion of the Adams spectral sequences in dimensions $\equiv$ $0 \bmod 4$ (which is all that is relevant for maps from $H P^{4 p+1}$ ) for $\Sigma P_{16 p-11}, \Sigma P_{16 p-9}$, $\Sigma P_{16 p-11} \wedge b o$, and $\Sigma P_{16 p-9} \wedge b o$, which correspond to the $k$-invariants for liftings to each of the spaces in (2.2).

By $[6,1.8], n H_{t}^{S p}$ lifts to $B^{\circ}(m)$ if and only if for all $i \leqslant t, \nu\left(\left(\begin{array}{c}n \\ i\end{array}\right)\right) \geqslant \nu\left(\pi_{4 i}\left(\Sigma P_{m} \wedge b o\right)\right)$. By standard methods, one finds that

$$
\nu\left(\left(\begin{array}{c}
-(4 p+2) \\
4 p+\varepsilon
\end{array}\right)\right)= \begin{cases}\alpha(p)-1, & \varepsilon=-2, \\
2+\alpha(p)+\nu(p), & \varepsilon=-1 \\
\alpha(p), & \varepsilon=0, \\
\alpha(p)+1, & \varepsilon=1 .\end{cases}
$$

We have $\alpha(p)=5$. Thus, $-(4 p+2) H_{4 p-1}^{S p}$ lifts to $B^{\circ}(16 p-11)$, and $-(4 p+2) H_{4 p}^{S p}$ lifts to $B^{\circ}(16 p-9)$. By considering the induced map of MPTs for $B^{\circ}(16 p-11) \rightarrow B^{\circ}(16 p-9)$, we deduce that $-(4 p+2) H_{4 p}$ lifts to $E_{5}$ in the MPT for $B^{\circ}(16 p-11)$. Then, since all 


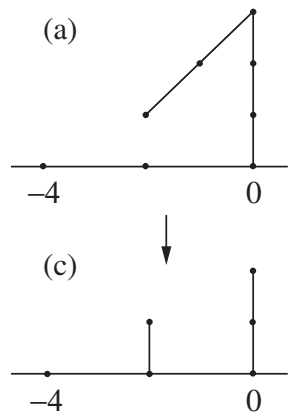

(b)

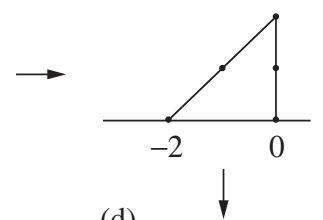

(d)

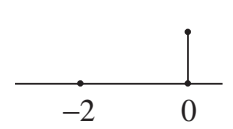

Figure 3. Some homotopy groups: (a) $\pi_{4 \ell+x}\left(\Sigma P_{4 \ell-5} \wedge b o\right)$; (b) $\pi_{4 \ell+x}\left(\Sigma P_{4 \ell-3} \wedge b o\right)$;

$$
\text { (c) } \pi_{4 \ell+x}\left(\Sigma P_{4 \ell-5} \wedge b u\right) ;(d) \pi_{4 \ell+x}\left(\Sigma P_{4 \ell-3} \wedge b u\right) \text {. }
$$

$k$-invariants for $\widetilde{\mathrm{BSp}}(16 p-11)$ which are relevant for $H P^{4 p}$ map injectively to those of $B^{\circ}(16 p-11)$, we may infer that $H P^{4 p}$ lifts to $E_{5}$ of the MPT for $\widetilde{\mathrm{BSp}}(16 p-11)$. We follow this into $E_{5}$ of the MPT for $\widetilde{\mathrm{BSp}}(16 p-9)$. Since this MPT has no $k$-invariants in dimension $16 p+4$ in filtration less than 5 , the map $H P^{4 p} \rightarrow E_{5}(16 p-9)$ extends over $H P^{4 p+1}$, establishing Proposition 2.1.

\section{Proof of Theorem 1.2}

Let $n=2 \ell$ with $\alpha(\ell)=3$. We must show that the map $C P^{2 \ell} \stackrel{f}{\rightarrow} B U \rightarrow B S O$ which classifies the stable normal bundle $-(2 \ell+1) H_{2 \ell}^{\mathbb{C}}$ factors through $B S O(4 \ell-4)$. The fibre in $P_{4 \ell-4} \rightarrow B S O(4 \ell-4) \rightarrow B S O$ has an ASS chart that looks like the first four dimensions of Figure 1 except that, if $\ell$ is even, twice the bottom class in what appears in that chart as dimension $16 p-4$ equals the sum of the two dots in the box above it. We will show that the map lifts to level 3 in the MPT for this fibration, and that the level- $3 k$-invariant is in primary indeterminacy, which implies that the lifting exists. The reason that we did not notice this result in [7] is apparently that we were hesitant to consider liftings to $B S O(m)$ when $m$ is divisible by 4 and the bundle is an odd multiple of the complex Hopf bundle. (See [7, Tables 1.8 and 1.9].)

We let $\widetilde{B U}(m)$ denote the classifying space for stably almost complex vector bundles of real geometric dimension $m$; i.e. it is the pull-back of $B U$ and $B O(m)$ over $B O$. As in [7], we use spaces $\widetilde{B U}(m) \rightarrow B_{m}^{\mathrm{o}} \rightarrow B_{m}^{\mathrm{u}}$ over $B U$ with fibres through dimension $2 m-2$ given by $P_{m} \rightarrow P_{m} \wedge b o \rightarrow P_{m} \wedge b u$. We need the charts of homotopy groups given in Figure 3.

We also need the easily obtained fact that, for $\varepsilon=0,1,2, \nu\left(\left(\begin{array}{c}-(2 \ell+1) \\ 2 \ell-\varepsilon\end{array}\right)\right)=3,2,2$, respectively. We use $[\mathbf{7}$, Theorem $1.7 \mathrm{~b}]$, which states that if $p$ is odd, then $p H_{n}^{\mathbb{C}}$ lifts to $B_{m}^{\mathrm{o}}$ if and only if, for all $i \leqslant n, \nu\left(\left(\begin{array}{l}p \\ i\end{array}\right)\right) \geqslant \nu\left(\pi_{2 i}\left(\Sigma P_{m} \wedge b u\right)\right)$, and, for all even $i \leqslant n$, $\nu\left(\left(\begin{array}{l}p \\ i\end{array}\right)\right) \geqslant \nu\left(\pi_{2 i}\left(\Sigma P_{m} \wedge b o\right)\right)$. This implies that our map $f: C P^{2 \ell} \rightarrow B U$ lifts to $B_{4 \ell-3}^{\text {o }}$, and $f \mid C P^{2 \ell-1}$ lifts to $B_{4 \ell-5}^{\mathrm{o}}$. Thus, $f$ lifts to level 3 in the MPT for $B_{4 \ell-5}^{\mathrm{o}}$. By [10, Tables 8.4, 8.12], for $* \leqslant 4 \ell, \pi_{*}\left(\Sigma P_{4 \ell-5}\right) \rightarrow \pi_{*}\left(\Sigma P_{4 \ell-5} \wedge b o\right)$ is surjective with kernel consisting of a single class in $*=4 \ell-1$. Since $H^{4 \ell-1}\left(C P^{2 \ell}\right)=0$, we conclude that $f$ lifts to level 3 
in the MPT for $\widetilde{B U}(4 \ell-5)$, and hence also in those for $\widetilde{B U}(4 \ell-4)$ and $B S O(4 \ell-4)$, using the maps of MPTs induced by $\widetilde{B U}(4 \ell-5) \rightarrow \widetilde{B U}(4 \ell-4) \rightarrow B S O(4 \ell-4)$.

We now employ a standard indeterminacy argument, as explained clearly in [15], to show that the final obstruction in $H^{4 \ell}\left(E_{3}\right)$ can be varied, if necessary. Let $E_{i}$ denote the spaces in the MPT of $B S O(4 \ell-4) \rightarrow B S O$. The fibre $F$ of $E_{3} \rightarrow E_{2}$ is $K_{4 \ell-4} \times$ $K_{4 \ell-2} \times K_{4 \ell-1} \times K_{4 \ell-1}$, corresponding to elements at height 2 in Figure 1, desuspended once. Here $K_{i}=K(\mathbb{Z} / 2, i)$. If $f_{3}: C P^{2 \ell} \rightarrow E_{3}$ sends the $k$-invariant $k_{3} \in H^{4 \ell}\left(E_{3}\right)$ trivially, then the lifting to $B S O(4 \ell-4)$ exists, since there are no more even-dimensional $k$-invariants. If $f_{3}^{*}\left(k_{3}\right) \neq 0$, then we will show that the composite

$$
C P^{2 \ell} \stackrel{\iota_{4 \ell-4} \times f_{3}}{\longrightarrow} F \times E_{3} \stackrel{\mu}{\rightarrow} E_{3},
$$

which is also a lifting of $f$, sends $k_{3}$ to 0 , and hence the lifting to $B S O(4 \ell-4)$ exists. Here $\mu$ denotes the action of the fibre on the total space in the principal fibration, and $\iota_{4 \ell-4}: C P^{2 \ell} \rightarrow F$ is the map which is non-trivial into the first factor of $F$. This will follow because a computation of the relations in the MPT, performed below, shows that

$$
\begin{aligned}
\mu^{*}\left(k_{3}\right)=1 \times k_{3}+\mathrm{Sq}^{1} \iota_{4 \ell-1}^{\prime} \times 1+\mathrm{Sq}^{2} \iota_{4 \ell-2} & \times 1+\iota_{4 \ell-2} \times w_{2} \\
& +\mathrm{Sq}^{4} \iota_{4 \ell-4} \times 1+\iota_{4 \ell-4} \times\left(w_{4}+w_{2}^{2}\right) .
\end{aligned}
$$

Thus, the composite (3.1) sends $k_{3}$ to

$$
f_{3}^{*}\left(k_{3}\right)+\mathrm{Sq}^{4}\left(x^{2 \ell-2}\right)+x^{2 \ell-2} \cdot\left(w_{4}+w_{2}^{2}\right)(-(2 \ell+1) H) .
$$

Here $x$ denotes the generator of $H^{2}\left(C P^{2 \ell}\right)$. Since $w_{2}(-(2 \ell+1) H)=x$, and $\mathrm{Sq}^{4}\left(x^{2 \ell-2}\right)$ and $w_{4}(-(2 \ell+1) H)$ are either both non-zero $(\ell$ even) or both zero $(\ell$ odd $)$, we deduce that $f_{3}^{*}\left(k_{3}\right)$ can be varied, if necessary, establishing the lifting.

We conclude the proof by listing in Table 3 the relations in the MPT of $B S O(4 \ell-4) \rightarrow$ $B S O$, the last of which yields the crucial fact (3.2). These relations are computed by the method initiated in $[\mathbf{8}]$ and used in $[\mathbf{7}, \mathbf{1 2}, \mathbf{1 5}]$. It is a matter of building a minimal resolution using Massey-Peterson algebras. In Table 3, $\varepsilon=1$ if $\ell$ is even, and $\varepsilon=0$ if $\ell$ is odd.

\section{Discussion of one of Crabb's proofs}

In $[\mathbf{3}$, Theorem 0.2], Crabb presented many new immersions of complex projective spaces. About his proof, he wrote 'Details will be omitted', although sketched arguments for each case were presented. We have checked the details of his arguments and found what appears to be a flaw in one case. This case was of particular interest to us, because, if true, it would have implied a new immersion result for real projective space which would be an addition to [5]. We present here our analysis of this case.

Proposition 4.1. The argument for the portion of [3, Theorem 0.2] which states that if $k$ is even and $\alpha(k)=4$, then $C P^{2 k+1}$ immerses in $\mathbb{R}^{8 k-6}$ is invalid. The question of whether this immersion exists is unresolved. 
Table 3. Relations in MPT for $B S O(4 \ell-4)$

\begin{tabular}{ll}
\hline$w_{4 \ell-3}$ & \\
$w_{4 \ell-2}$ & \\
$w_{4 \ell}$ & \\
\hline$k_{4 \ell-3}^{1}:$ & $\mathrm{Sq}^{1} w_{4 \ell-3}$ \\
$k_{4 \ell-2}^{1}:$ & $\left(\mathrm{Sq}^{2}+w_{2}\right) w_{4 \ell-3}$ \\
$k_{4 \ell-1}^{1}:$ & $\left(\mathrm{Sq}^{2}+w_{2}\right) w_{4 \ell-2}$ \\
$k_{4 \ell}^{1}:$ & $\mathrm{Sq}^{1} w_{4 \ell}+\left(\mathrm{Sq}^{2}+w_{2}\right) \mathrm{Sq}^{1} w_{4 \ell-2}$ \\
$\tilde{k}_{4 \ell}^{1}:$ & $\varepsilon \mathrm{Sq}^{1} w_{4 \ell}+\left(\mathrm{Sq}^{4}+w_{4}\right) w_{4 \ell-3}+\left(w_{2} \mathrm{Sq}^{1}+w_{3}\right) w_{4 \ell-2}$ \\
\hline$k_{4 \ell-3}^{2}:$ & $\mathrm{Sq}^{1} k_{4 \ell-3}^{1}$ \\
$k_{4 \ell-1}^{2}:$ & $\left(\mathrm{Sq}^{2}+w_{2}\right) k_{4 \ell-2}^{1}+\left(\mathrm{Sq}^{3}+w_{3}\right) k_{4 \ell-3}^{1}$ \\
$k_{4 \ell}^{2}:$ & $\mathrm{Sq}^{1} k_{4 \ell}^{1}+\left(\mathrm{Sq}^{2}+w_{2}\right) k_{4 \ell-1}^{1}$ \\
$\tilde{k}_{4 \ell}^{2}:$ & $\mathrm{Sq}^{1} \tilde{k}_{4 \ell}^{1}+\left(\mathrm{Sq}^{2} \mathrm{Sq}^{1}+w_{3}\right) k_{4 \ell-2}^{1}+\left(\mathrm{Sq}^{4}+w_{4}+w_{2}^{2}+w_{2} \mathrm{Sq}^{2}\right) k_{4 \ell-3}^{1}$ \\
\hline$k_{4 \ell-3}^{3}:$ & $\mathrm{Sq}^{1} k_{4 \ell-3}^{2}$ \\
$k_{4 \ell}^{3}:$ & $\mathrm{Sq}^{1} \tilde{k}_{4 \ell}^{2}+\left(\mathrm{Sq}^{2}+w_{2}\right) k_{4 \ell-1}^{2}+\left(\mathrm{Sq}^{4}+w_{4}+w_{2}^{2}\right) k_{4 \ell-3}^{2}$ \\
\hline & \\
\hline
\end{tabular}

Proof. Let $k=2 K$. To obtain the immersion, one must prove that $-(4 K+2) H_{4 K+1}^{\mathbb{C}}$ lifts to $\widetilde{\mathrm{BSp}}(8 K-8)$. The $K O$-theoretic obstruction for lifting this bundle, calculated similarly to that in $\S 1$, is 0 . However, there are several elements in the kernel of the reduction from the total obstruction to the $K O$-theoretic obstruction which cannot be ruled out. In [2, Table 4.1], these are the three $\mathbb{Z} / 2$ summands in row 1 , column 9 . In our Figure 1, which, after a slight re-indexing, presents the obstructions for this lifting question as well as the lifting question considered in $\S 1$, these correspond to three of the many dots in column $16 p+2$.

Crabb realized that these could cause a problem, and so he hoped to use the factorization through $H P^{2 K}$, in a similar method to that in $\S 1$. In fact, he wrote in his proof (on p. 166) that in several cases, including this one, it can be shown that the bundle over the quaternionic projective space is stably equivalent to a bundle of the desired dimension. In this case, he would be saying that $-(2 K+1) H_{2 K}^{S p}$ lifts to $\widetilde{\mathrm{BSp}}(8 K-8)$. However, this lifting does not exist; its $K O$-theoretic obstruction is non-zero.

This can be seen by a calculation similar to that in $\S 1$. We use [3, Proposition 2.5]. With $\lambda(T)$ as in $\S 1$, the necessary condition is that there exists an integer $e$ such that $e\left(\lambda^{4 K-1}\right)_{2} \equiv 16 \bmod 32, e\left(\lambda^{4 K-1}\right)_{1} \equiv 8 \bmod 16$, and $e\left(\lambda^{4 K-1}\right)_{0} \equiv 0 \bmod 2$. Since

$$
\left(1-\frac{1}{3} T+\frac{8}{45} T^{2}\right)^{4 K-1}=1+\mathrm{od} \cdot T+\mathrm{od} \cdot T^{2}
$$

$\bmod \left(T^{3}\right)$, where 'od' denotes an odd fraction, we require $e$ to satisfy both $e \equiv 16 \bmod 32$ and $e \equiv 8 \bmod 16$, which is clearly impossible.

We close by commenting on the relationship between Crabb's necessary conditions for immersion involving powers of $\lambda(T)=\left(\sinh ^{-1}(\sqrt{T}) / \sqrt{T}\right)^{2}$, which we have used above, and the Sigrist-Suter necessary condition involving powers of $\log (1+t) / t$ in Theorem 1.4. 
These conditions involving power series can be directly related to one another by a slight extension of [1, Theorem 1.5], which was proved using a proof suggested to the authors by Crabb, and based on his earlier topological work described in $[\mathbf{4}, \S 4]$.

\section{Evaluation of a Sigrist-Suter condition}

In Theorem 1.4, a general statement of a necessary condition for $C P^{n}$ to immerse in $\mathbb{R}^{4 n-2 \alpha(n)}$ is presented. We evaluate this explicitly when $\alpha(n)=8$ in the following result, which we have incorporated into Tables 1 and 2.

Proposition 5.1. If $\alpha(n)=8$ and $C P^{n}$ immerses in $\mathbb{R}^{4 n-2 \alpha(n)}$, then $\nu(n-7)=3$ or $\nu(n-6)=4$ or $\nu(n-5)=5$ or $\nu(n-4)=7$.

This follows readily from Theorem 1.4 and the following lemma.

Lemma 5.2. Let $(\log (1+t) / t)^{m}=\sum c_{m, i} t^{i}$. Then

$$
\nu\left(c_{m, 7}\right)=\nu\left(c_{m, 8}\right)<\nu\left(c_{m, i}\right) \text { for all } i<7
$$

if and only if $\nu(m-7)=4$ or $\nu(m-5)=5$ or $\nu(m-3)=6$ or $\nu(m-1)=8$.

Proof. Let

$$
v(m)=\left(v_{0}(m), \ldots, v_{8}(m)\right)=\left(\nu\left(c_{m, 0}\right), \ldots, \nu\left(c_{m, 8}\right)\right) .
$$

Additionally, define a function, for which some values are only specified to satisfy an inequality, by

$$
\nu(k, e) \begin{cases}=\nu(k) & \text { if } \nu(k)<e, \\ \geqslant e & \text { if } \nu(k) \geqslant e .\end{cases}
$$

The lemma follows immediately from the following, which we will prove for $v(m)$ by induction on $m$ (note that some components are only asserted to satisfy an inequality):

$$
\begin{aligned}
& \text { if } e \geqslant 3 \text {, then } v\left(2^{e}(2 a+1)\right)=(0, e-1, e-3, e-3, e-6, e-5, e-7, e-7, e-11) \text {; } \\
& v(7+8 k)=(0,-1,-2,-3,-4,-5,-6,-7,-8+\nu(k, 2)) ; \\
& v(5+8 k)=(0,-1,-1, \geqslant-1,-4,-5, \geqslant-4,-6,-8+\nu(k, 3)) ; \\
& v(3+32 k)=(0,-1,-2,-3, \geqslant-1,-4,-4,-5,-6+\nu(k, 2)) \\
& v(19+32 k)=(0,-1,-2,-3,-2,-4, \geqslant-3, \geqslant-4,-7) \text {; } \\
& v(11+16 k)=(0,-1,-2,-3,-3, \geqslant-3,-5,-6,-8) \text {; } \\
& v(9+16 k)=(0,-1, \geqslant-1,-2,-3,-4,-4, \geqslant-3,-8) \text {; } \\
& v(17+32 k)=(0,-1,0,-2,-2,-3,-3, \geqslant-2,-7) \text {; } \\
& v(1+32 k)=(0,-1,0,-2, \geqslant-1, \geqslant-2, \geqslant-2,-3,-6+\nu(4, k)) .
\end{aligned}
$$

We begin by using MAPLE to verify $v(m)$ for $m=8,7,5,3,19,11,9,17,1$. The induction proof for $v\left(2^{e}\right)$ is obtained by using

$$
\sum c_{2^{e+1}, i} t^{i}=\left(\sum c_{2^{e}, i} t^{i}\right)^{2}
$$


We have

$$
v_{i}\left(2^{e+1}\right)=1+v_{i}\left(2^{e}\right)
$$

for $1 \leqslant i \leqslant 8$, since, as is easily verified, the right-hand side of (5.1) is strictly less than $1+v_{j}\left(2^{e}\right)+v_{i-j}\left(2^{e}\right)$ for $1 \leqslant j \leqslant \frac{1}{2} i$.

Next we obtain the claim for $v\left(2^{e}(2 a+1)\right)$ from $\left(\sum c_{2^{e}, i} t^{i}\right)\left(\sum c_{2^{e+1} a, i} t^{i}\right)$. From this, we obtain $v_{i}\left(2^{e}(2 a+1)\right)=v_{i}\left(2^{e}\right)$, since the strict minimum of $v_{j}\left(2^{e}\right)+v_{i-j}\left(2^{e+1} a\right)$ is obtained when $j=i$, which is essentially the same verification as the previous one.

The cases in which the asserted $v_{i}\left(u+2^{e} k\right)$ is not of the form ' $\geqslant t$ ' or $\nu(k, t)$ are, except for $v_{7}(1+32 k)$ with $k$ odd, obtained from $v_{i}(u)+v_{0}\left(2^{e} k\right)$, which, in these cases, is strictly less than other values of $v_{j}(u)+v_{i-j}\left(2^{e} k\right)$. Quite a few verifications are required for this. For example, since $v_{4}\left(2^{e} k\right)=e+\nu(k)-6$, for $e \geqslant 3$, it is relevant that

$$
v_{i}(u)<v_{i-4}(u)- \begin{cases}3, & u=7,5 \\ 2, & u=11,9 \\ 1, & u=3,19,17,1\end{cases}
$$

in the cases in which the asserted value of $v_{i}(u)$ is a single integer. These cases are differentiated according to whether the argument of $v$ is $u+8 k, u+16 k$ or $u+32 k$. If $k$ is odd, then $v_{7}(1+32 k)=-3$ comes from having each of $v_{7}(1)+v_{0}(32 k), v_{3}(1)+v_{4}(32 k)$ and $v_{1}(1)+v_{6}(32 k)$ equal to -3 .

In the cases in which the asserted $v_{i}\left(u+2^{e} k\right)$ is of the form ' $\geqslant t$ ', one verifies, unless $i=7$ and $u=9$ or 17 , that $v_{j}(u)+v_{i-j}\left(2^{e} k\right) \geqslant t$ for all $j$, with the possibility of equality for several values of $j$. For example, $v_{6}(5+8 k) \geqslant-4$ comes from $v_{6}(5)+v_{0}(8 k) \geqslant-4$, $v_{4}(5)+v_{2}(8 k) \geqslant-4$ and $v_{2}(5)+v_{4}(8 k) \geqslant-4$, while $v_{j}(5)+v_{6-j}(8 k)>-4$ for $j=0,1,3,5$. If $k$ is odd, the exceptional case $v_{7}(9+16 k) \geqslant-3$ comes from $v_{1}(9)+v_{6}(16 k)=-4$ and $v_{3}(9)+v_{4}(16 k)=-4$, with other values greater than or equal to -3 . The case $v_{7}(17+32 k)$ is similar.

Finally, for $\nu(k)=0$ or $1, v_{8}(7+8 k)=-8+\nu(k)$ comes from $v_{0}(7)+v_{8}(8 k)$, which is strictly less than all other $v_{j}(7)+v_{8-j}(8 k)$ while, if $\nu(k) \geqslant 2$, we have $v_{0}(7)+v_{8}(8 k)=$ $\nu(k)-8$ and $v_{8}(7)+v_{0}(8 k) \geqslant-6$, with other terms larger. The same argument works for $v_{8}(5+8 k), v_{8}(3+32 k)$ and $v_{8}(1+32 k)$.

\section{References}

1. M. BENDERSKY AND D. M. DAVIS, The unstable Novikov spectral sequence for $S p(n)$, and the power series $\sinh ^{-1}(x)$, London Mathematical Society Lecture Notes Series, Volume 176, pp. 73-86 (Cambridge University Press, 1992).

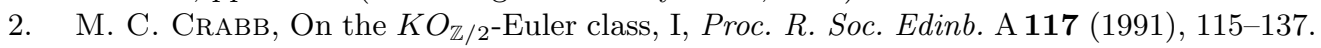

3. M. C. CRABb, Immersing projective spaces in Euclidean space, Proc. R. Soc. Edinb. A 117 (1991), 155-170.

4. M. C. CRABb And K. Knapp, James numbers, Math. Annln 282 (1988), 395-422.

5. D. M. DAVIs, Table of immersions and embeddings of real projective spaces (available at www.lehigh.edu/ dmd1/immtable).

6. D. M. DAVIS AND M. Mahowald, The geometric dimension of some vector bundles over projective spaces, Trans. Am. Math. Soc. 205 (1975), 295-315. 
7. D. M. DAVIS AND M. MAHOWALD, Immersions of complex projective spaces and the generalized vector field problem, Proc. Lond. Math. Soc. 35 (1977), 333-348.

8. S. Gitler And M. Mahowald, The geometric dimension of real stable vector bundles, Bol. Soc. Mat. Mex. 11 (1966), 85-107.

9. I. M. James, Some embeddings of projective spaces, Proc. Camb. Phil. Soc. 55 (1959), 294-298.

10. M. Mahowald, The metastable homotopy of $S^{n}$, Memoirs of the American Mathematical Society, Volume 72 (American Mathematical Society, Providence, RI, 1967).

11. A. Mukherjee, Embedding complex projective spaces in Euclidean space, Bull. Lond. Math. Soc. 13 (1981), 323-324.

12. A. D. Randall, Some immersion theorems for projective spaces, Trans. Am. Math. Soc. 147 (1970), 135-151.

13. B. J. SAnderson And R. Schwarzenberger, Nonimmersion theorems for differential manifolds, Proc. Camb. Phil. Soc. 59 (1963), 312-322.

14. F. Sigrist AND U. Suter, On immersions of $C P^{n}$ in $\mathbb{R}^{4 n-2 \alpha(n)}$, Lecture Notes in Mathematics, Volume 673, pp. 106-115 (Springer, 1978).

15. N. Singh, On nonimmersion of real projective spaces, Topol. Applic. 136 (2004), 233-238.

16. B. SteER, On immersing complex projective $(4 k+3)$-space in Euclidean space, $Q . J$. Math. 22 (1971), 339-345. 